\title{
Breast Cancer in the 21st Century: Neu Opportunities and Neu Challenges
}

\author{
Stuart J. Schnitt, M.D. \\ Department of Pathology, Beth Israel Deaconess Medical Center and Harvard Medical School, Boston, \\ Massachusetts
}

Recent advances in the understanding of the molecular and genetic alterations underlying breast cancer development and progression have provided the opportunity to develop novel therapeutic strategies for this disease. None of these developments has had a greater recent impact on clinicians and pathologists than the recognition of the importance of the HER-2/neu (c-erbB-2) oncogene. Located on chromosome 17, this gene encodes a 185 kD transmembrane glycoprotein with tyrosine kinase activity that functions as a growth factor receptor. Amplification or overexpression of HER-2/neu is seen in approximately 20 to $30 \%$ of invasive breast cancers and this has been considered to be an adverse prognostic factor in many studies. However, recent interest in HER-2/neu has largely been focused on its role as a potential target for breast cancer treatment. In particular, recognition of the role of HER2/neu in breast cancer growth led to the development of a humanized monoclonal antibody directed against the HER-2/neu protein as a therapeutic agent (Herceptin). Clinical studies have further suggested that HER-2/neu status can provide important information regarding sensitivity to certain forms of conventional systemic therapy, particularly anthracyclines. As a result of these developments, there has been increasing demand for pathologists to perform assays for HER-2/neu on current and archived breast cancer specimens. Immunohistochemistry and fluorescence in situ hybridization have emerged as the most viable assays for evaluation of HER-2/neu in routine clinical practice. However, each of these methods has its advantages and disadvantages. Determining the rel-

Copyright () 2001 by The United States and Canadian Academy of Pathology, Inc.

VOL. 14, NO. 3, P. 213, 2001 Printed in the U.S.A.

Date of acceptance: December 12, 2000.

Address reprint requests to: Stuart J. Schnitt, M.D., Department of Pathology, Beth Israel Deaconess Medical Center, East Campus, 330 Brookline Ave., Boston, MA 02215; e-mail: sschnitt@caregroup.harvard.edu; fax: 617-975-5620. ative merits of these assays and developing clinically meaningful and reproducible systems to report the results are challenges pathologists must now address.The development of a therapeutic agent that directly targets a protein involved in a growth-signaling pathway represents a new paradigm in breast cancer treatment. Therapeutic strategies that target other molecules involved in breast cancer development and progression are on the horizon. It is crucial that pathologists become aware of these advances and assume a pivotal role in the development and application of assays to evaluate these new molecular targets.

KEY WORDS: Breast cancer, HER2/neu, erb-2, Prognosis, Immunohistochemistry, Fluorescence in situ hybridization.

Mod Pathol 2001;14(3):213-218

The traditional approaches to treating breast cancer include surgery, radiation therapy, chemotherapy and hormonal therapy. Although these therapeutic modalities, singly and in various combinations, are effective in many patients, they do not specifically target the tumor.

Recent advances in the understanding of the molecular and genetic alterations underlying breast cancer development and progression have initiated a paradigm shift in the treatment of breast cancer. In this new paradigm, the development of new treatments for breast cancer will result from the identification of specific molecular targets that are discovered in studies designed to elucidate the genes and molecules involved in breast tumorigenesis (1-3). This in turn will result in the identification of molecularly defined patient subgroups. Of particular importance to pathologists, the integration of diagnostic tests and therapeutics will be a critical element in this scenario.

None of the recent developments in the understanding of the molecular events underlying breast tumorigenesis has had a greater immediate impact 
on both clinicians and pathologists than the recognition of the importance of the HER2/neu (cerbB-2) oncogene in breast cancer $(4,5)$. In particular, the recent development of a therapeutic agent that directly targets the HER2 protein (Herceptin, trastuzamab) represents a model for the future of breast cancer treatment directed toward a specific molecular target.

\section{BACKGROUND}

The HER2/ neu gene is the human analog of the rat neu gene identified in rat neuroblastomas in the early 1980s (reviewed in Refs. 4-8). This gene has been found to be amplified and/or overexpressed in approximately 25 to $30 \%$ of invasive breast cancers in humans, most commonly in invasive ductal carcinomas. Located on chromosome 17q21, the HER2/neu gene encodes a $185 \mathrm{kD}$ transmembrane glycoprotein with tyrosine kinase activity that functions as a growth factor receptor. This protein is a member of the epidermal growth factor receptor family of receptor tyrosine kinases, which includes epidermal growth factor receptor, HER2, HER3, and HER4. Ligand binding to one of these receptors results in the formation of homodimers and heterodimers. Dimerization is followed by phosphorylation, which, in turn, results in a cascade of downstream signaling events that are important for cell growth and maintenance of the transformed state. Of note, although HER2 forms heterodimers with other members of the family on interaction with their ligands, it has no known ligand of its own and is therefore considered an orphan receptor. However, at very high levels of overexpression, HER2 may undergo spontaneous homodimerization, which can initiate downstream signaling, stimulating cell growth and maintaining cellular transformation. This may be clinically important in HER2overexpressing tumors.

\section{CLINCIAL SIGNIFICANCE OF HER2/NEU AMPLIFICATION AND OVEREXPRESSION}

\section{HER2/Neu as a Prognostic Factor (reviewed in refs. 5,9$)$}

A prognostic factor is one that provides information regarding patient outcome at the time of diagnosis. In 1987, Slamon and colleagues were the first to report a relationship between HER2 and prognosis in patients with breast cancer. These investigators found that amplification of this gene was associated with significantly reduced survival in a group of patients with node-positive disease. Since that time, there have been numerous studies assessing the prognostic significance of HER2 amplification and overexpression in patients with breast cancer.

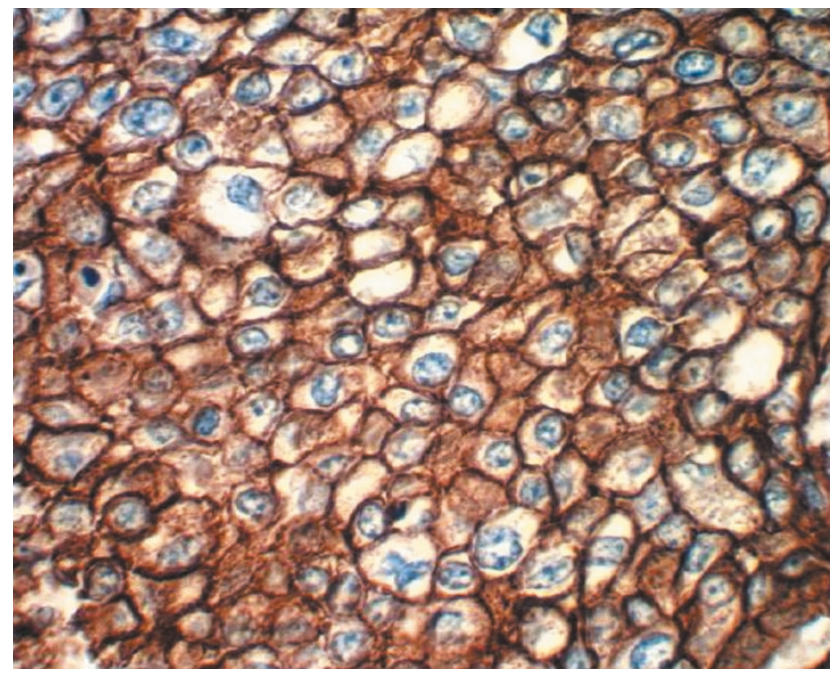

FIGURE 1. Immunohistochemistry for HER2 protein. This case shows intense circumferential tumor cell membrane staining for HER2 protein and is readily categorized as positive for HER2 overexpression. Almost all cases with this level of immunostaining show HER2 gene amplification.

Most studies have shown that HER2 amplification and overexpression is a significant adverse prognostic factor in patients with node-positive disease. However, the prognostic significance in patients with node-negative breast cancer is much more controversial with approximately equal numbers of studies indicating that this either is or is not an independent prognostic factor. There are many issues that have contributed to this controversy including small patient numbers in many of the studies, patient selection, variations in treatment, variations in length of follow-up, variations in statistical analysis, and variations in methodology used to assess HER2 status. Because HER2 overexpression has been reported to be associated with other adverse prognostic factors such as positive lymph nodes, larger tumor size, high histologic grade, high proliferation rate, and lack of expression of estrogen and progesterone receptors, some studies have shown that HER2 is prognostically important in univariate analysis but loses its significance in multivariate analysis. Nonetheless, the weight of evidence suggests that HER2 overexpression is associated with an adverse clinical outcome in patients with breast cancer. Whether it is a dependent factor or an independent factor is probably less important than understanding the biologic explanation for this association.

\section{HER2/Neu as a Predictive Factor (reviewed in refs. 5, 9)}

A predictive factor is one that provides information regarding the likelihood of response to a given therapeutic modality. In recent years, there has been great interest in the interactions between HER2 overexpression and various forms of systemic 


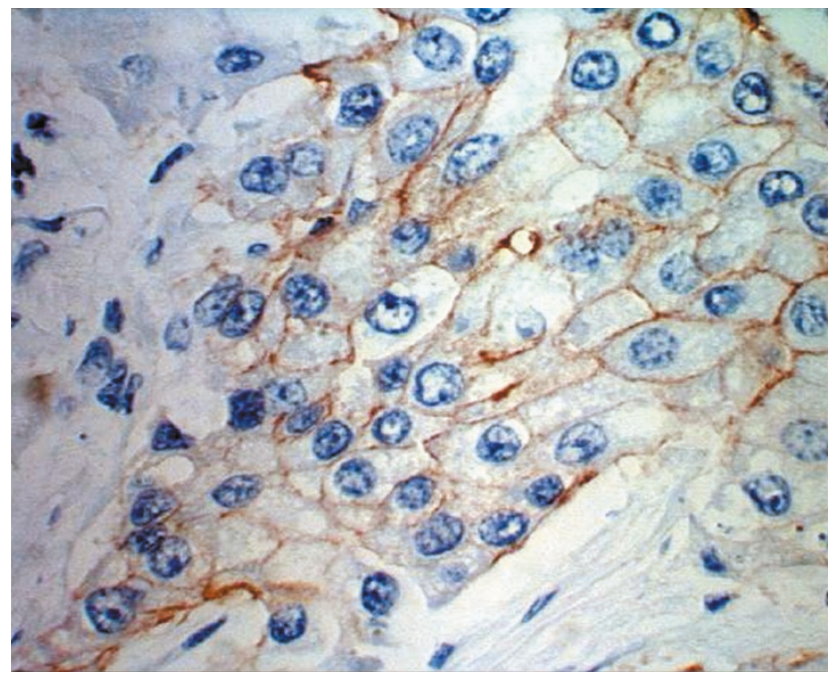

FIGURE 2. Immunohistochemistry for HER2 protein. In this case, there is weak membrane staining around some of the tumor cells. Cases such as this are subject to considerable differences in interpretation. Only a minority of cases with this level of HER2 staining show HER2 gene amplification.

therapy in patients with breast cancer because this could provide information that helps in the determining the most suitable systemic therapeutic regimen in a given patient. The most compelling evidence of such an interaction has been seen in patients treated with chemotherapeutic regimens containing anthracyclines. These studies have generally shown that HER2 overexpression is associated with increased sensitivity to anthracyclines. Although some studies have indicated that HER2 overexpression is associated with resistance to cyclophosphamide, methotrexate and 5-fluorouracil (CMF) chemotherapy, others have not. Similarly studies evaluating the interaction between HER2 overexpression and taxanes have variously shown both resistance to and sensitivity to this cytotoxic agent. Finally, some studies have indicated that HER2 overexpression is associated with tamoxifen resistance whereas others have not found such an association. In summary, there is fairly consistent evidence that HER2 overexpression is predictive of sensitivity to anthracyclines. Although patients with HER2 overexpressing tumors may be somewhat less responsive to $\mathrm{CMF}$ and to tamoxifen than those with tumors that do not show HER2 overexpression, the data are insufficient to deny patients treatment with either CMF or tamoxifen based on the HER2 status of the tumor.

\section{HER2/Neu as a Therapeutic Target}

HER2 represents an ideal therapeutic target because it is functionally important in breast cancer growth, it is accessible as a cell surface receptor, it is expressed at high levels in breast tumors, and it is expressed at low levels in normal tissue (reviewed

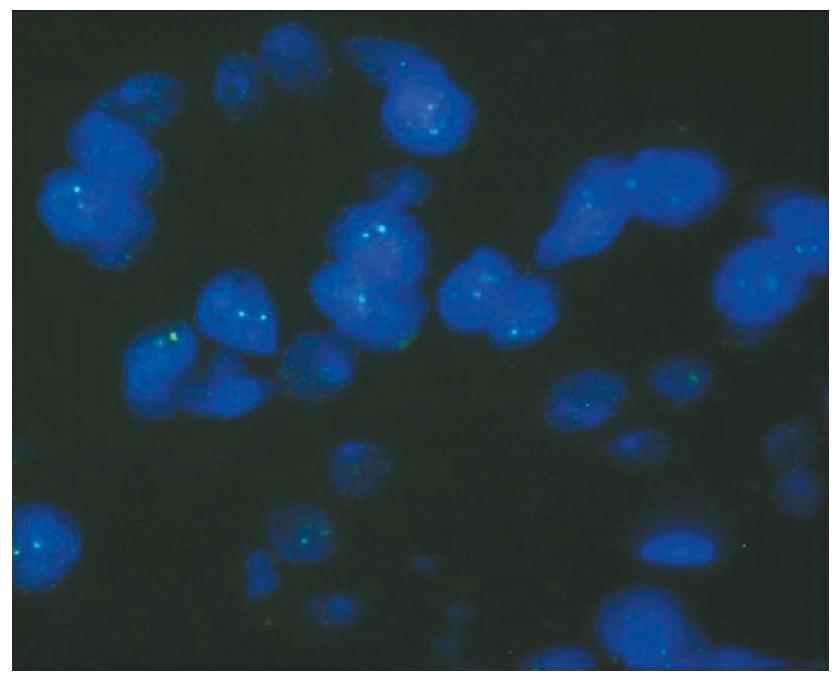

FIGURE 3. Fluorescence in situ hybridization for HER2 gene amplification. In this case, only one or two fluorescent signals are present in each tumor cell nucleus, indicating that the HER2 gene is not amplified.

in Refs. 5, 9, 10). Preclinical studies showed that monoclonal antibodies directed against HER2 were capable of inhibiting both the in vitro proliferation of HER2 overexpressing tumor cells and the in vivo growth of HER2 overexpressing human breast cancer xenografts in nude mice. These experiments set the stage for using an antibody to HER2 as a therapeutic agent to treat patients with breast cancer. However, it was not until scientists at Genentech developed a humanized monoclonal antibody to HER2 that it became feasible to use this strategy to treat patients. This antibody, known as Herceptin (trastuzamab), has been shown to be active as a single agent in the treatment of patients with metastatic breast cancer who failed treatment with prior chemotherapy (11). Furthermore, results of a randomized clinical trial have demonstrated that the combination of Herceptin plus chemotherapy was more effective than chemotherapy alone as first-line treatment in the metastatic setting (12). It should be noted, however, that the likelihood of response to Herceptin in this trial was related to the level of HER2 overexpression. These studies have further demonstrated that Herceptin is fairly welltolerated. The most important side effect is cardiac toxicity, which is most common in patients who received concurrent or prior anthracyclines ( 25 to $30 \%)$ (13). Although this level of toxicity may considered acceptable in patients with metastatic disease, it is of greater concern if Herceptin is to be used in the adjuvant setting. The beneficial effect of Herceptin in the clinical studies conducted to date has been modest. However, its activity in patients with HER2-overexpressing tumors is proof of the biologic principle that a therapeutic agent directed toward a specific molecular target can be used to treat patients with cancer. 


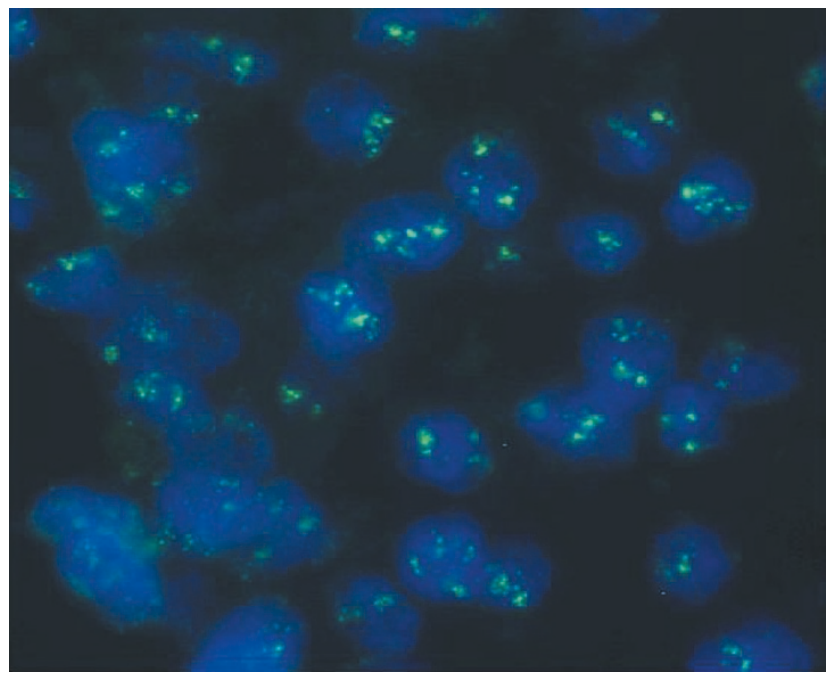

FIGURE 4. Fluorescence in situ hybridization for HER2 gene amplification. Numerous fluorescent signals are present in each nucleus of this case, indicating amplification of the HER2 gene.

There are many unresolved issues regarding the role of Herceptin as a therapeutic agent. Its role as a single agent and in combination with chemotherapy needs to be further defined. The role of Herceptin in the adjuvant setting is an important clinical question that is currently being evaluated in clinical trials as is its role in patients with breast cancers that do not overexpress HER2. The role of Herceptin in the treatment of other cancers that overexpress HER2 is also being evaluated. Finally, there is an active effort to develop other treatments targeting HER2 or its downstream effector molecules, and vaccines against HER2 are currently being studied.

\section{ASSESSMENT OF HER2 STATUS IN PATIENTS WITH BREAST CANCER}

There is currently great clinical demand for determining the HER2 status of current and archived breast cancer cases. Unfortunately, this clinical demand has outpaced our ability to develop a single, reliable, reproducible test that fulfills all of the clinical needs. Although there are a variety of different methods to assess HER2 status of breast cancers, the two that are the most practical to perform in the routine practice of pathology are immunohistochemistry (IHC) to assess HER2 protein overexpression and fluorescence in situ hybridization (FISH) to assess HER2 gene amplification $(4,9,14-16)$ (Figs. 1-4). Each of these methods has its advantages and disadvantages, as indicated in Table 1.

Studies directly comparing IHC and FISH in the same cases have demonstrated overall concordance rates of approximately 80 to $95 \%$ (17). Concordance between these two methods is excellent for cases that are either completely negative or strongly pos-
TABLE 1. Pros and Cons of Immunohistochemistry and Fluorescence In Situ Hybridization for Determining HER2 Status in Breast Cancer

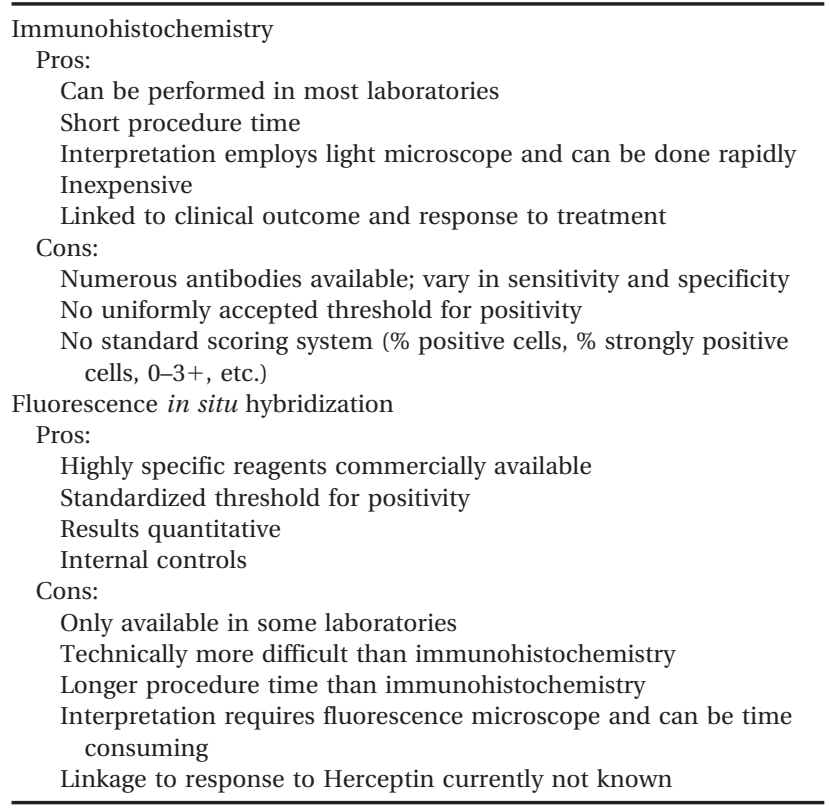

itive by IHC. In contrast, only a minority of cases that show weak staining by IHC show gene amplification by FISH $(\sim 10-30 \%)$. The relative clinical importance of IHC and FISH with regard to assessing prognosis and predicting response to chemotherapy and Herceptin is not yet clear, and this is currently under active investigation. Furthermore, the clinical and biologic significance of discordant cases merits further study.

Unfortunately, at the present time, there is considerable confusion among pathologists and clinicians with regard to which method and which reagents are most appropriate to use for HER2 testing in routine clinical practice $(4,9,14-17)$. There are currently three FDA-approved assays for HER2 evaluation (Table 2). However, no one assay is FDAapproved for all clinical purposes (i.e., assessing prognosis, predicting response to standard chemotherapy, and determining suitability for Herceptin treatment). Furthermore, for any given patient, the clinical use of HER2 assay results may change over time. For example, at the time of diagnosis, clinicians may use information regarding the HER2 status to predict the likelihood of response of a given patient to anthracyclines or to help assess progno-

TABLE 2. FDA-Approved Assays for HER2

\begin{tabular}{|c|c|c|}
\hline Test & Type & Indication \\
\hline Hercep Test (Dako) & IHC & Suitability for Herceptin \\
\hline Path Vysion (Vysis) & FISH & Assessing prognosis \\
\hline & & $\begin{array}{l}\text { Predicting response to } \\
\text { standard chemotherapy }\end{array}$ \\
\hline Inform (Oncor/Ventana) & FISH & Assessing prognosis \\
\hline
\end{tabular}

IHC, immunohistochemistry; FISH, fluorescence in situ hybridization. 


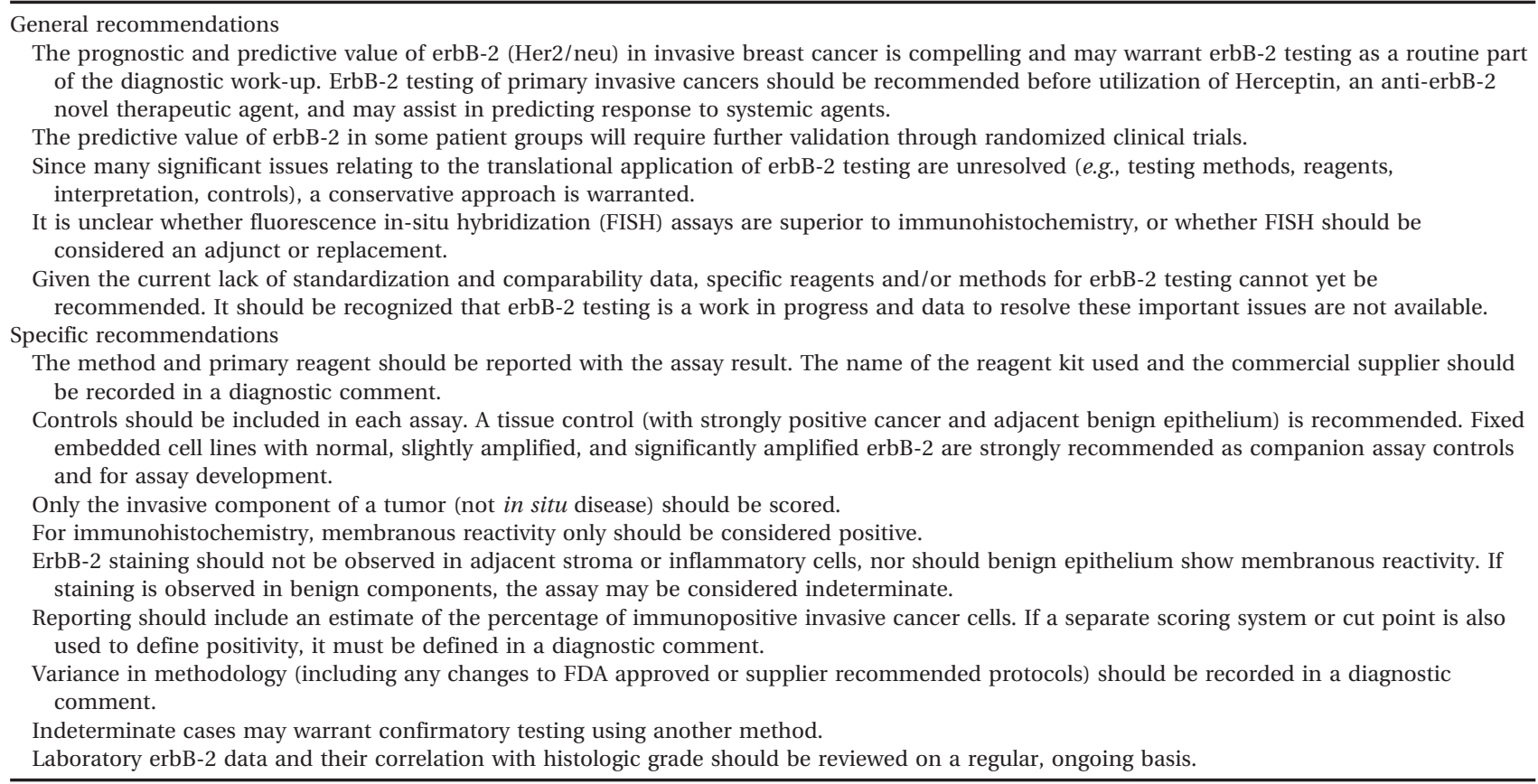

sis. However, when that same patient develops metastatic disease some time after their initial diagnosis, the HER2 status may be used to determine that patient's suitability for Herceptin treatment. Finally, FDA-approval does not obligate pathologists to use that particular test.

The College of American Pathologists has recently issued recommendations regarding HER2 testing for patients with breast cancer (18). These are summarized in Table 3 . These recommendations largely reflect the current lack of consensus with regard to how best to evaluate HER2 status.

\section{IMPLICATIONS FOR THE FUTURE}

Although the role of HER2 as a prognostic factor and as a factor predicting response to conventional systemic therapy has been widely discussed, the ultimate importance of the HER2 story is likely to be that it represents a model for cancer treatment using a therapeutic agent directed toward a specific molecular target. The HER2-Herceptin story is certain to be only the first of many examples of molecularly targeted treatment of breast cancer. In fact, there is a pressing clinical need to develop a comprehensive profile of the biologic and molecular characteristics of a tumor, rather than assessing one marker at a time. In the near future, the tools of molecular biology, such as microarray technology $(19,20)$, will permit such an assessment and will dramatically alter the manner in which tumors are classified and prognostic and predictive factors are determined. For example, it is conceivable that in the future, after a biopsy of a breast cancer is per- formed, RNA will be isolated from the tumor cells, and the RNA (or cDNA) applied to a microarray to determine the gene expression pattern of the tumor. A computerized profile of this gene expression pattern will then be generated and provide the molecular fingerprint of the tumor with regard to behavior, resistance or responsiveness to various therapeutic modalities, and biologic targets for intervention. However, the new molecular biology methods will need to be integrated with standard methods of pathologic evaluation. Indeed, determining how best to integrate routine pathologic evaluation with newer, molecular-based methods is the most immediate challenge to pathologists as we enter the 21st century.

\section{NOTE ADDED IN PROOF}

Recent data, presented in abstract form, have suggested that HER2 gene amplification detected by FISH may be a better predictor of response to Herceptin than HER2 protein overexpression detected by immunohistochemistry (Mass $\mathrm{R}$, et al. The concordance between the clinical trials assay [CTA] and fluorescence in situ hybridization [FISH] in the Herceptin pivotal trials. Presented at the Annual Meeting of the American Society of Clinical Oncology, May 2000).

\section{REFERENCES}

1. Perez E. Current management of metastatic breast cancer. Semin Oncol 1999;26(Suppl. 12):1-10. 
2. Hortobagyi GN, Hung M-C, Buzdar A. Recent developments in breast cancer therapy. Semin Oncol 1999;26(Suppl. 12):11-20

3. Borden EC, Esserman L, Linder DJ, Campbell MJ, Fulton AM. Biological therapies for breast carcinoma: Concepts for improvement in survival. Semin Oncol 1999;26(Suppl. 12):28-40.

4. DiGiovanna MP. Clinical significance of HER-2/neu overexpression: part I. PPO Updates 1999;13(9):1-10.

5. DiGiovanna MP. Clinical significance of HER-2/neu overexpression: part II. PPO Updates 1999;13(10):1-14.

6. Reese DM, Slamon DJ. HER-2/neu signal transduction in human breast and ovarian cancer. Stem Cells 1997;15:1-8.

7. Hung M-C, Lau Y-K. Basic science of HER-2/neu: a review. Semin Oncol 1999;26(Suppl. 12):51-9.

8. Slikowski MX, Lofgren JA, Lewis GD, Hotaling TE, Fendly BM, Fox JA. Non-clinical studies addressing the mechanism of action of trastuzamab (Herceptin). Semin Oncol 1999; 26(Suppl. 12):60-70.

9. Ross JS, Fletcher JA. HER-2/neu (c-erbB-2) gene and protein in breast cancer. Am J Clin Pathol 1999;112(Suppl. 1):S53-67.

10. Shak S, for the Herceptin Multinational Investigator Study Group. Overview of the trastuzamab (Herceptin) anti-HER2 monoclonal antibody clinical program in HER2-overexpressing metastatic breast cancer. Semin Oncol 1999; 26(Suppl. 12):71-7.

11. Cobleigh MA, Vogel CL, Tripathy D, et al. Efficacy and safety of Herceptin (humanized anti-HER2 antibody) as a single agent in 222 women with HER2 overexpression who relapsed following chemotherapy for metastatic breast cancer [Abstract]. Proc Am Soc Clin Oncol 1998;17: 97a.

12. Slamon DJ, Leyland-Jones, Shak S, et al. Addition of Herceptin (humanized anti-HER2 antibody) to first line chemother- apy for HER2 overexpressing metastatic breast cancer markedly increases anticancer activity: A randomized multinational controlled phase III trial [Abstract]. Proc Am Soc Clin Oncol 1998;17:98a.

13. Ewer MS, Gibbs HR, Swafford J, Benjamin RS. Cardiotoxicity in patients receiving trastuzamab (Herceptin): primary toxicity, synergistic or sequential stress or surveillance artifact. Semin Oncol 1999;26(Suppl. 12):96-101.

14. Mitchell MS, Press MF. The role of immunohistochemistry and fluorescence in situ hybridization for HER-2/neu in assessing the prognosis of breast cancer. Semin Oncol 1999; 26(Suppl. 12):108-16.

15. Ravdin PM. Should HER2 status be routinely measured for all breast cancer patients? Semin Oncol 1999;26(Suppl. 12): 117-23.

16. Hanna W, Kahn HJ, Trudeau M. Evaluation of HER-2/neu status in breast cancer: From bench to bedside. Mod Pathol 1999;12:827-34.

17. Jacobs TJ, Gown AM, Yaziji H, Barnes MJ, Schnitt SJ. Comparison of fluorescence in situ hybridization and immunohistochemistry for the evaluation of HER2/neu in breast cancer. J Clin Oncol 1999;17:1974-8.

18. Fitzgibbons PL, Page DL, Weaver D, et al. Prognostic factors in breast cancer: College of American Pathologists Consensus Statement 1999. Arch Pathol Lab Med 2000;124:966-78.

19. Duggan DJ, Bittner M, Chen Y, Meltzer P, Trent JM. Expression profiling using cDNA microarrays. Nature Genet 1999; 21(Suppl.):10-4.

20. Brown PO, Botstein D. Exploring, the new world of the genome with DNA microarrays. Nature Genet 1999; 21(Suppl.):33-7. 